\title{
Ordinary Disc Herniation Changing into Posterior Epidural Migration of Lumbar Disc Fragments Confirmed by Magnetic Resonance Imaging: A Case Report of a Successful Endoscopic Treatment
}

\author{
Kiyoshi Tarukado, Osamu Tono, Toshio Doi \\ Department of Orthopedic Surgery, Kyusyu University Beppu Hospital, Oita, Japan
}

\begin{abstract}
The posterior epidural migration of lumbar disc fragments is an extremely rare event with an unknown pathogenesis. To the best of our knowledge, there are no previously reported cases of a change of ordinary disc herniation into the posterior epidural migration of lumbar disc fragments as confirmed by magnetic resonance imaging (MRI). A 26-year-old male presented to our department complaining of left buttock and lateral leg pain. An ordinary herniation was shown in the first MRI. The patient's unilateral symptoms changed into bilateral symptoms while awaiting admission to the hospital. Posterior migrated lumbar disc fragments were shown in the second MRI taken at the time of admission. Microendoscopic surgery providing a detailed observation of the region was performed. Our case indicates that an ordinary lumbar disc herniation may lead to the posterior migration of lumbar disc fragments, and that microendoscopic surgery may provide a treatment.
\end{abstract}

Keywords: Lumbar; Hernia; Endoscopy

\section{Introduction}

Lombardi [1] reported the first case of posterior epidural migrated lumbar disc fragments in 1973. Although disc fragments are known to migrate to superior, inferior or lateral sites in the anterior epidural space [2-4], the posterior epidural migration of lumbar disc fragments is extremely rare. Posterior epidural migrated lumbar disc fragments are often confused with other posterior epidural space-occupying lesions $[2,5,6]$; and the differential diagnosis of these epidural lesions includes cysts, abscesses, tumors and hematomas. In all previously reported cases, magnetic resonance imaging (MRI) at the first visit showed posterior epidural lesions. To the best of our knowledge, there are no reported cases of a change of ordinary disc herniation into posterior epidural migration of lumbar disc fragments confirmed by MRI. In the present report, we obtained images in the hyperacute phase of the posterior epidural migration of lumbar disc fragments. Our study is also unique for its successful microendoscopic discectomy treatment of the posterior epidural migrated lumbar disc fragments, which has not been reported in the literature. The pathogenesis of posterior epidural migrated lumber disc fragments is unknown. In the present case, a detailed observation in the hyperacute phase was possible, and the patient was

Received Mar 28, 2013; Revised Sep 5, 2013; Accepted Oct 11, 2013

Corresponding author: Kiyoshi Tarukado

Department of Orthopedic Surgery, Kyusyu University Beppu Hospital,

4546 Tsurumihara, Tsurumi Beppu, Oita 874-0838, Japan

Tel: +81-977-27-1600, Fax: +81-977-27-1755, E-mail: kt99059@hotmail.com 
successfully treated by microendoscopic surgery. The findings of this case could help to clarify the mechanisms underlying the posterior epidural migration.

\section{Technical Note}

A 26-year-old male presented to our department complaining of left buttock and lateral leg pain. His patellar tendon reflex (PTR) and Achilles tendon reflex (ATR) were normal, femoral nerve stretch test (FNST) was normal, and straight leg raising test (SLRT) was positive for his left leg. Hypesthesia of the L5 dermatome on the left side and 4 of 5 strength on the dorsiflexion in the left foot and abduction of the left leg were revealed during his first visit to the hospital. Plain radiographs revealed a reduction of the disc height in the L4-5 and L5-S1 discs. MRI showed an extruded disc, compressing the thecal sac para-centrally on the left side at the L4-5 level. The lesion exhibited iso-intensity on T1-weighted MRI and low intensity on T2-weighted MRI (Fig. 1). No right leg symptoms were present. We diagnosed the patient with left L5 radiculopathy due to the lumber disc herniation at L4-5. The visual analog scale (VAS) of the left leg was $100 \mathrm{~mm}$, and left leg palsy was seen. The patient returned home, and was scheduled to undergo surgery at a later date. However, he developed right leg pain after lifting a heavy load three days before his scheduled admission. When admitted to the hospital one week after the first visit, the patient complained of bilateral buttock and leg pain. Hypesthesia of the L5 dermatome on the bilateral sides and a 4 of 5 strength on the dorsiflexion in the left foot and abduction of the left leg were revealed. His PTR was normal, bilateral ATR was diminished, FNST was normal

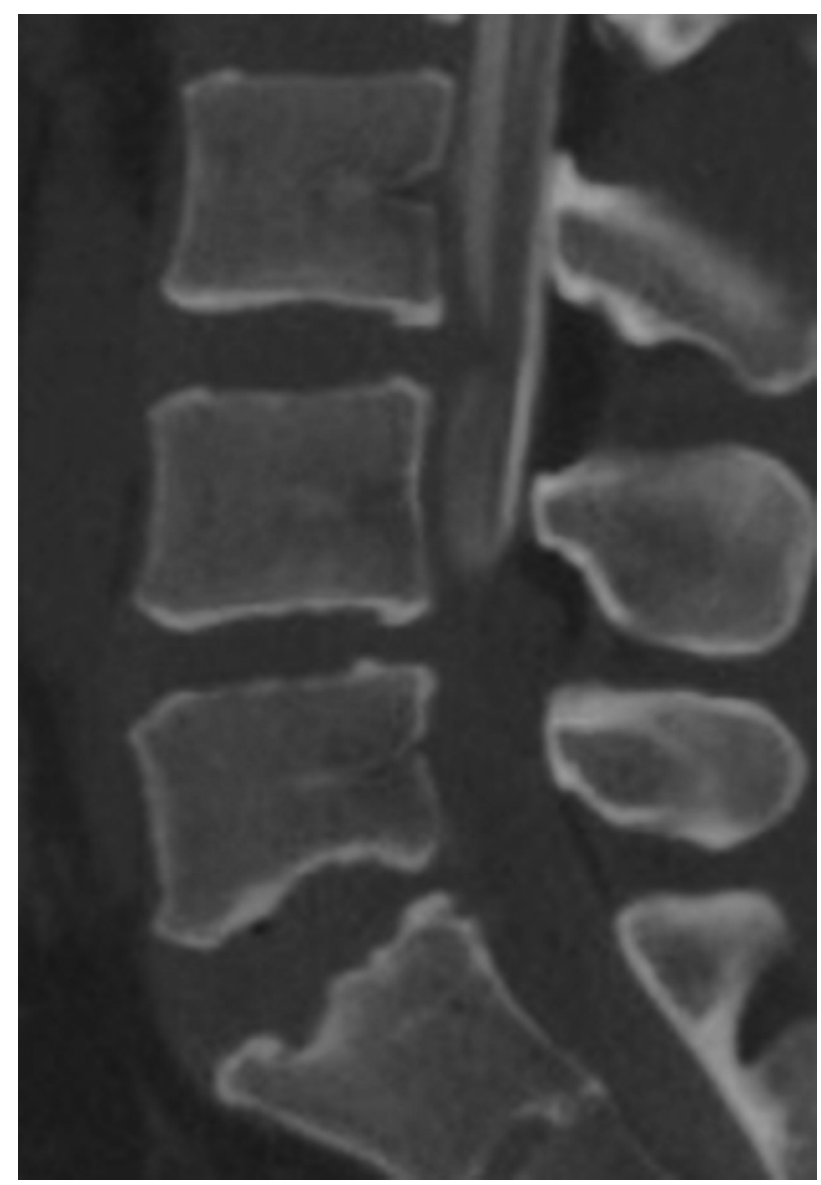

Fig. 2. Myelography showed a complete block at the L4-5 level.


Fig. 1. Ordinary lumbar disc herniation was observed on the left side at the L4-5 level. (A) Ordinary lumbar disc herniation was observed at L4-5 on sagittal T2-weighted magnetic resonance imaging (MRI). (B) Ordinary lumbar disc herniation was observed at L4-5 on sagittal T1-weighted MRI. (C) Ordinary lumbar disc herniation was observed on the left side at L4-5 on axial T2-weighted MRI. 
and SLRT was positive on the left leg. Myelography was performed, and showed a complete block at L4-5 (Fig. 2). A mass-like lesion was found on the posterior epidural space on the left side. The lesion exhibited iso-intensity on T1-weighted MRI and low intensity on T2-weighted MRI (Fig. 3).

Posterior epidural space-occupying lesions must generally be distinguished from many differential diagnoses,
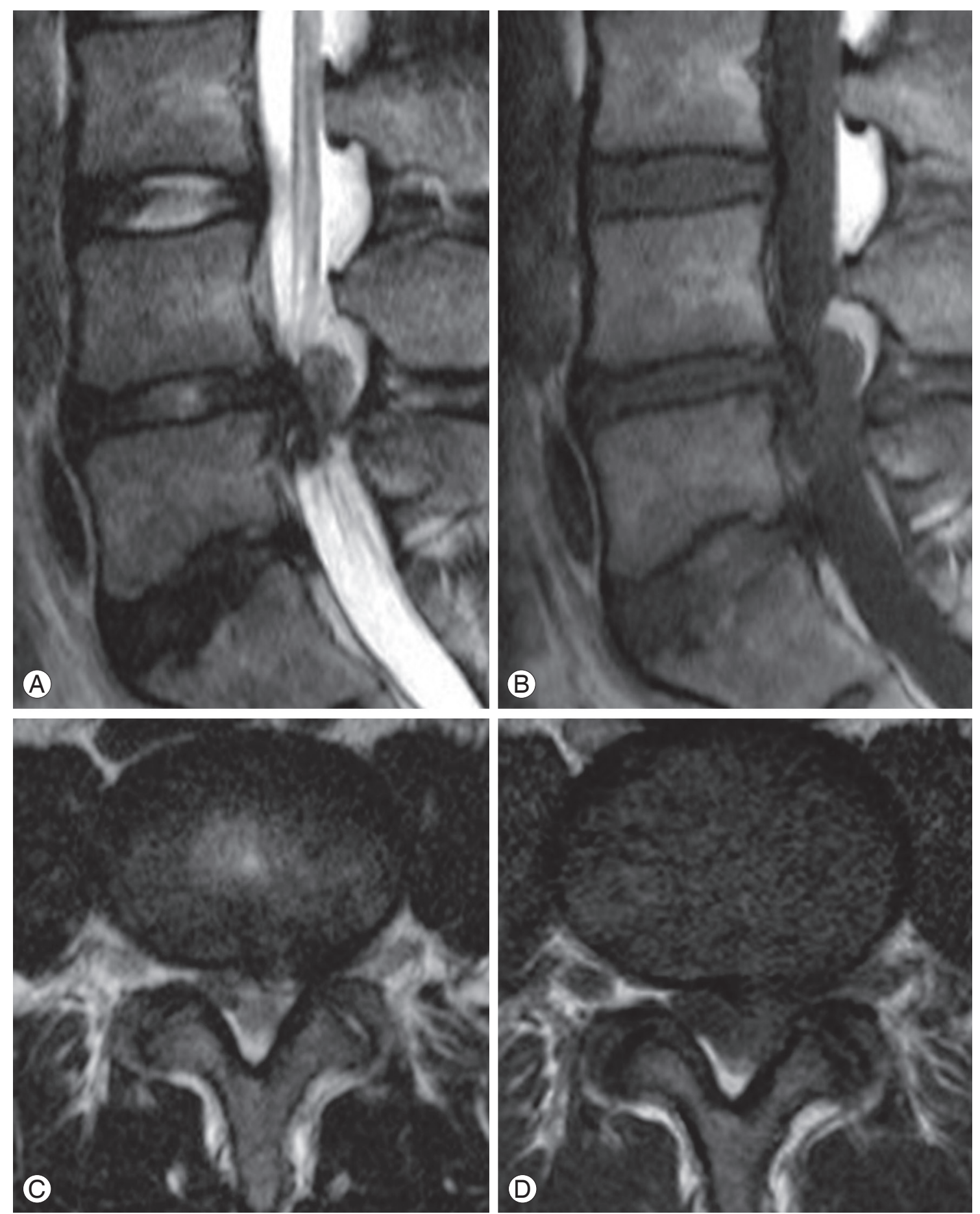

Fig. 3. T1- and T2-weighted magnetic resonance imaging (MRI) showed the lesion in the posterior epidural space at L4-5. (A) Sagittal T2-weighted MRI showed the lesion in the posterior epidural space at L4-5. (B) Sagittal T1-weighted MRI showed the lesion in the posterior epidural space at L4-5. (C) Axial T2-weighted MRI showed the lesion in the posterior epidural space on the left side at L4-5. (D) Axial T1-weighted MRI showed the lesion in the posterior epidural space on the left side at L4-5. 
such as cysts, abscesses, tumors and hematomas. However, in the present case, ordinary herniation was confirmed in the first MRI. The signal intensities in the second MRI were the same as those during the first MRI. The period between the first and second MRI was one week. Therefore, the mass-like lesion was diagnosed as the posterior epidural migration of lumbar disc fragments, as it was attached to the L4-5 disc. Microendoscopic surgery was performed to treat the patient. When freeing the flavum from the superior articular process on the left side, a huge herniation emerged from the epidural space (Fig. 4). The huge disc fragment had been extruded into the posterior epidural space and wrapped around from the outside of the nerve root. After removal of the disc fragment, the nerve root and the rent in the annulus seemed to be stretched more than usual (Fig. 5). No adhesion was seen. With the surgery completed, an adequate decompression of the nerve root was confirmed. His VAS score was rapidly improved after the surgery. The palsy of the



Fig. 4. Herniated nucleus pulpous (HNP) existed posterior to the flavum. dorsiflexion in the foot and abduction of the leg also recovered. The results of his neurological assessments were satisfactory, and the VAS score was improved at the final follow-up examination one year after the surgery.

\section{Discussion}

We herein reported a case of an ordinary disc herniation that changed into a posterior epidural migration of lumbar disc fragments, as confirmed by MRI. We defined posterior migrated lumbar disc fragments as below. Most of the herniated nucleus pulpous (HNP) existed behind, across the center of dural sac on MRI. To date, several cases of the posterior epidural migration of lumbar disc fragments have been reported [1-3,5,6]. However, to the best of our knowledge, the MRI at the time of the first visit showed posterior epidural lesions in all previously reported cases. In the previous reports, the posterior epidural migrated lumbar disc fragments tended to demonstrate a variety of intensity signals on T1- and T2-weighted images. The features of these images were unrelated to the duration of symptoms prior to the time they were taken. This suggests that some cases develop symptoms as soon as the posterior epidural migration occurs, while in other cases, the symptoms are secondary factors that develop after the migration into the posterior epidural space. The features of the imaging findings may depend on the absorption process. In the present case, we were able to obtain MR imaging findings of the posteriormigrated lumbar disc fragments in the hyperacute stage, and the features of the imaging findings were the same as those of ordinary disc herniation.

The pathomechanism(s) of posterior epidural migra-
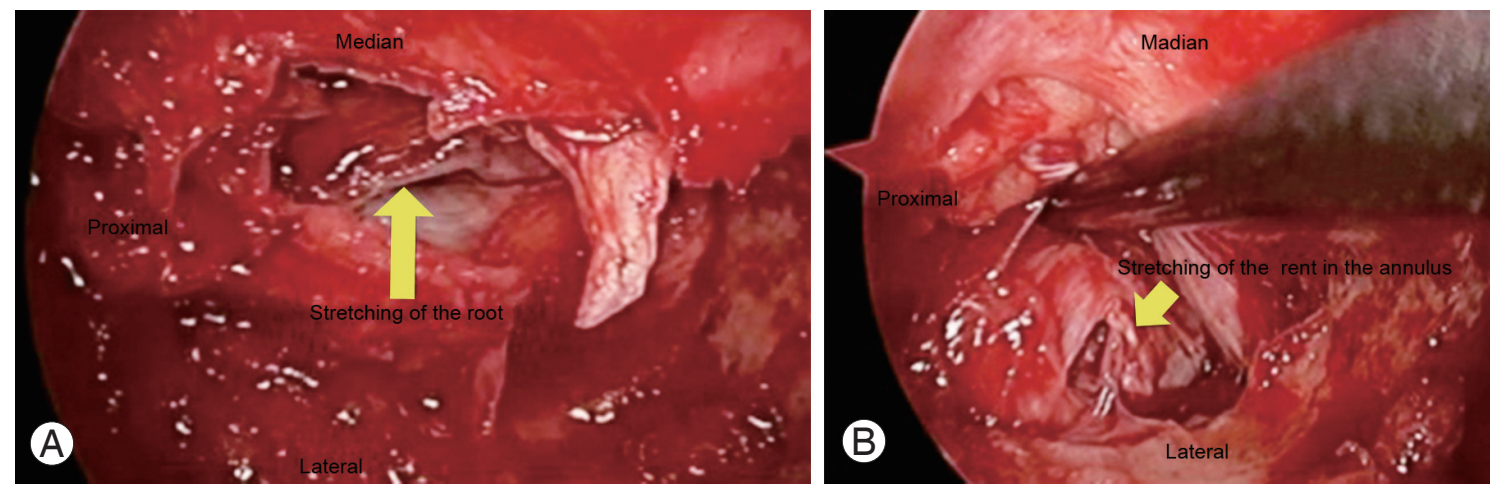

Fig. 5. Stretching of the nerve root and the rent in the annulus were observed. (A) Stretching of the nerve root more than usual was observed after removal of the disc fragments. Arrow indicates the stretched left L5 nerve root. (B) Stretching of the rent in the annulus more than usual was observed after removal of the disc fragments. Arrow indicates the stretched rent in the annulus. 
tion remains unknown. It is generally considered that the anatomical barrier has been disturbed at some point prior to the posterior epidural migration. In particular, the peridural membrane, epidural fat, epidural venous plexus and the nerve root itself presumably act as impediments to posterior migration, and must be affected to allow for the migration to occur [2,3,7]. In the present case, a deflection of the rent in the annulus and the nerve root were observed after removal of the disc fragments. It is expected that a fairly high pressure would be required to accomplish such a deflection. We speculated that the nerve root may have functioned as an inhibitor of the migration due to the deflection.

Microendoscopic surgery has been applied for various conditions, including herniation, for the excellent visualization of the spinal canal it provides. Furthermore, microendoscopic surgery allows for the minimally invasive removal of epidural lesions [8,9]. This technique can be an effective alternative to conventional open surgery for epidural lesions in the lumbar spine. Microendoscopic surgery was chosen for these reasons in our case.

To the best of our knowledge, our case is the first reported case of the treatment of posterior epidural migrated lumbar disc fragments by microendoscopic discectomy. The operation plan was made as below. HNP existed behind, on the left side of dural sac on MRI. We judged that microendoscopic discectomy was possible. If it was difficult to remove the HNP through the microendoscopic discectomy, we were going to perform microendoscopic laminotomy through the unilateral approach and remove HNP. The posterior migrated lumbar disc fragments were successfully treated by microendoscopic discectomy, and the surgery was also able to provide a detailed observation of the intraoperative findings. Therefore, microendoscopic surgery may be considered for the surgical treatment of various epidural lesions, including the posterior epidural migrated lumbar disc fragments.

\section{Conflict of Interest}

No potential conflict of interest relevant to this article was reported.

\section{References}

1. Lombardi V. Lumbar spinal block by posterior rotation of anulus fibrosus: case report. J Neurosurg 1973;39:642-7.

2. Bonaroti EA, Welch WC. Posterior epidural migration of an extruded lumbar disc fragment causing cauda equina syndrome: clinical and magnetic resonance imaging evaluation. Spine (Phila Pa 1976) 1998;23:378-81.

3. Kuzeyli K, Cakir E, Usul H, et al. Posterior epidural migration of lumbar disc fragments: report of three cases. Spine (Phila Pa 1976) 2003;28:E64-7.

4. Schellinger D, Manz HJ, Vidic B, et al. Disk fragment migration. Radiology 1990;175:831-6.

5. Sengoz A, Kotil K, Tasdemiroglu E. Posterior epidural migration of herniated lumbar disc fragment. J Neurosurg Spine 2011;14:313-7.

6. Akhaddar A, El-Asri A, Boucetta M. Posterior epidural migration of a lumbar disc fragment: a series of 6 cases. J Neurosurg Spine 2011;15:117-28.

7. Dosoglu M, Is M, Gezen F, Ziyal MI. Posterior epidural migration of a lumbar disc fragment causing cauda equina syndrome: case report and review of the relevant literature. Eur Spine J 2001;10:348-51.

8. Ishii K, Matsumoto M, Watanabe K, Nakamura M, Chiba K, Toyama Y. Endoscopic resection of cystic lesions in the lumbar spinal canal: a report of two cases. Minim Invasive Neurosurg 2005;48:240-3.

9. Ohba T, Ebata S, Ando T, Ichikawa J, Clinton D, Haro $\mathrm{H}$. Lumbar ligamentum flavum hematoma treated with endoscopy. Orthopedics 2011;34:e324-7. 